\title{
Gas flow through microtubes with different internal surface coatings
}

\author{
M. Hadj Nacer, ${ }^{\text {a) }}$ I. Graur, P. Perrier, and J. G. Méolans \\ Aix-Marseille Université, CNRS, IUSTI UMR 7343, 5 rue Enrico Fermi, 13013 Marseille, France \\ M. Wuest \\ INFICON Ltd., Alte Landstrasse 6, LI-9496 Balzers, Liechtenstein
}

(Received 2 May 2013; accepted 21 October 2013; published 8 November 2013)

\begin{abstract}
An experimental setup based on the constant volume technique is developed to measure the mass flow rate through microtubes under isothermal stationary flow conditions. Four different working gases (helium, nitrogen, argon, and carbon dioxide), and two surface materials (stainless steel and Sulfinert) are considered. The Knudsen number calculated for the experimental conditions varies from $\sim 10^{-4}$ (hydrodynamic regime) to $\sim 5$ (transitional regime). In the reduced range $\left(10^{-4}-0.1\right)$ corresponding to the hydrodynamic and slip regimes, an approach based on the analytical solution of the Stokes equation subjected to a first order velocity slip boundary condition is used. The velocity slip coefficient and the tangential momentum accommodation coefficient are extracted from the experimental data of the mass flow rate using their analytical expressions. The results are summarized in the tables representing the accommodation coefficients for the corresponding gas-surface material combinations. The influence of the molecular mass on the tangential momentum accommodation coefficient is discussed. In addition, an original technique is proposed to extract the accommodation coefficient in the whole experimentally available range $\left(10^{-4}-5\right)$ of the gas rarefaction. The accommodation coefficients obtained using this technique are close to those found in the slip regime. (C) 2014 American Vacuum Society. [http://dx.doi.org/10.1116/1.4828955]
\end{abstract}

\section{INTRODUCTION}

The information about the properties of the gas-surface interaction such as condensation, adsorption, adhesion, and accommodation is important in vacuum technology. One of the characteristics of the gas-surface interaction is the accommodation coefficient. When a gas particle interacts with a surface, the energy is transferred in the form of heat and stress. This leads to two main types of accommodation called energy accommodation and tangential momentum accommodation. Accommodation is the process whereby the particles impinging a solid surface acquire its temperature and velocity. For isothermal flows, only tangential momentum accommodation needs to be considered. The tangential momentum accommodation coefficient (TMAC) denotes a momentum flux ratio characterizing the gas-surface interaction. This is the ratio between the tangential momentum flux transferred to the wall and the maximal tangential momentum flux occurring when the reflected particles are completely accommodated with the surface. In the frame of the Maxwellian kernel, the value of the TMAC varies in the range from 0 to 1 . In the case of a specular reflection, the tangential momentum of gas particles is maintained. This means that a gas molecule reflects from the surface with the same tangential velocity and the same absolute value of the normal velocity, but with opposite sign, and the TMAC is equal to 0 . In the case of diffuse reflection, the gas particles lose all of their tangential momentum and leave the surface with a Maxwellian distribution of the surface parameters. This case corresponds to a TMAC of 1 . The value of the accommodation coefficient depends on various parameters such as the surface properties and the weight of the gas

${ }^{a)}$ Electronic mail: mustafa.hadjnacer@polytech.univ-mrs.fr particle. Some other scattering kernels exist. ${ }^{1,2}$ In the Cercignani-Lampis scattering model, ${ }^{1}$ the TMAC may vary from 0 to 2 , where a value of 2 corresponds physically to the back scattering. However, in practice, the tangential momentum accommodation coefficient varies in a small range, from 0.7 to $1 .^{3}$ Thus, for the present isothermal study, the Maxwellian kernel is more simple and sufficiently realistic and will be exclusively used to describe the boundary conditions.

The experimental apparatus used in this work consists of a cylindrical microtube inserted between two reservoirs maintained at the same temperature. This setup allows us to obtain isothermal flows and to measure the corresponding stationary mass flow rates. In addition, the choice of microtube is theoretically and practically very interesting. Indeed, various effects of the interaction between the gas flow and the solid surface are increased by decreasing the conduit diameter owing to the increase in the surface-tovolume ratio. On the other hand, the conduits with small diameters are very useful in various domains, notably for gas chromatography and the mass spectrometry. Therefore, knowledge about gas-surface interaction properties becomes theoretically very important and is of great practical interest.

For isothermal gas flows, the gas-surface interaction is essentially characterized by the TMAC. The fraction of the tangential momentum transferred between the gas molecules and the surface has an impact on the macroscopic flow parameters, such as the bulk velocity and the mass flow rate. It is notable that, in the case of flow through a cylindrical tube, the mass flow rate may increase when a gas particle does not accommodate completely to the internal tube surface. 
The TMAC is required in expressions of the boundary conditions in the slip regime for continuum modeling and in all flow regimes for kinetic modeling of the reflection law. Therefore, a better knowledge of the mechanism of the gassolid surface interaction will allow us to improve the understanding of the gas flow behavior in the slip, transitional, and free molecular flow regimes, and to better design the industrial devices which work under rarefaction conditions.

Many researchers carried out different experiments to determine the TMAC values for various gas-solid surface pairs. The main experimental techniques used to measure the TMAC are reviewed in Ref. 4. This review shows that generally the accommodation coefficient depends upon a large number of parameters, such as the type of gas, the surface material, and its cleanliness and roughness.

The four main experimental techniques proposed in the literature to measure the mass flow rate through the microchannels are as follows: ${ }^{4}$ First is the liquid drop method, ${ }^{5-9}$ which permits a direct determination of the volume flow rate by tracking the interface of an oil drop moving in a calibrated tube as a function of time with a speed camera.

Second is the dual tank accumulation method, developed by Arkilic et al., ${ }^{10}$ which consists of measuring the relative pressure variation between a reference and an accumulation tank. The mass flow rate is deduced from the ideal gas law.

Third is the constant volume (CV) technique, , $^{5,9,11-15}$ which is similar to the dual tank accumulation method, but the absolute pressure variation in the tanks is measured. This approach needs very high thermal stability.

Last is the constant pressure technique. ${ }^{16-18}$ In contrast to the constant volume technique this method is not commonly used because of the difficulties in measuring the volume variation as a function of time while maintaining a constant pressure.

All of these methods have their own advantages and disadvantages. The simplest and the most accurate technique, however, is the CV technique, and is the technique adopted in the present study.

The mass flow rates of: helium $(\mathrm{He})$, nitrogen $\left(\mathrm{N}_{2}\right)$, argon (Ar), and carbon dioxide $\left(\mathrm{CO}_{2}\right)$ are measured under isothermal stationary flow conditions. Two long tubes of small diameter (several hundred microns) made from stainless steel (SS) are used, one of which has its internal surface covered by Sulfinert. The different flow regimes, hydrodynamic, slip, and transitional, are considered. In the slip flow regime, the velocity slip and accommodation coefficients are deduced explicitly using the mass flow rate experimental data and the Navier-Stokes model, subject to a first order slip boundary condition. In addition, for all of the considered flow regimes, the measured data are compared with the results of numerical simulation valid for all flow regimes. In this way, the accommodation coefficients are obtained using an original technique.

\section{EXPERIMENT}

\section{A. Methodology}

The experimental setup is represented schematically in Fig. 1. This setup was built to measure the mass flow rate

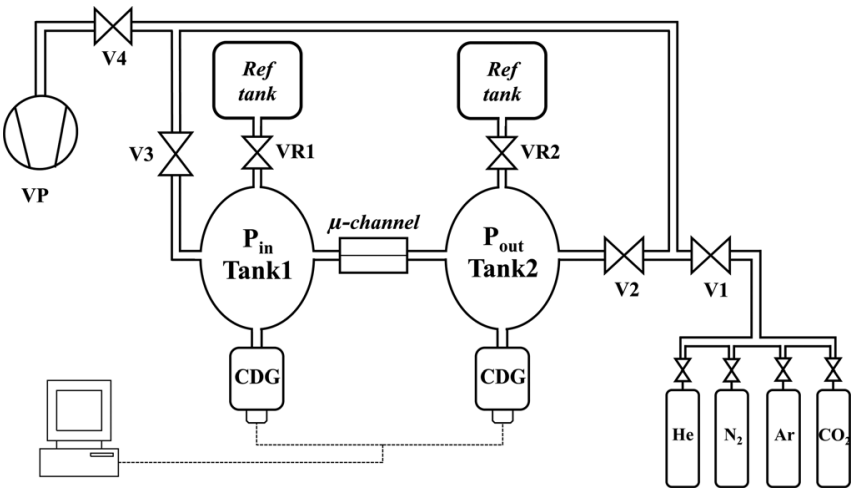

FIG. 1. Schematic of the experimental setup.

through the microtubes using the $\mathrm{CV}$ technique. The $\mathrm{CV}$ technique consists of measuring, as the function of time, the pressure variation between two constant volume tanks connected with a microchannel. The flow through the microchannel was generated by setting a pressure difference between the inlet and outlet tanks. The volume of the tanks must be much greater than the microchannel volume to guarantee that the flow parameters are independent of time, but must still remain detectable. The time-dependent pressure and temperature variations were measured and the mass flow rate was deduced from the equation of state. The mass variations occurring in the tanks during the experiments did not contradict the stationary assumption because the pressure variations were maintained at less than $2 \%$ during the experiments.

Leakage and outgassing tests were carried out with pressure sensors and are described in detail in Refs. 12 and 13.

The experimental procedure for the mass flow rate measurements is described as follows:

First, the experimental loop (Fig. 1) was evacuated using the vacuum pump by opening the valves $V 2, V 3$, and $V 4$ for $1-2 \mathrm{~h}$.

Next, the valve $V 4$ was closed and the system was filled with working gas from the high pressure tank by opening the valve $V 1$ until the desired inlet pressure value $\left(p_{\text {in }}\right)$ was reached.

The valves $V 1$ and $V 3$ were then closed, and the valve $V 4$ was opened again to decrease the pressure in the outlet tank until the desired value $\left(p_{\text {out }}\right)$ was obtained.

Finally, the valves $V 2$ and $V 4$ were closed, and the pressure and temperature data in both tanks were acquired.

The registration of the pressure variations in the inlet and outlet tanks was carried out using two capacitance diaphragm gauges (INFICON), as shown in Fig. 1. These were chosen for their pressure range and were connected to the upstream and downstream tanks.

The temperature of the gas inside the system was measured by three temperature sensors (PT100), which provided an accuracy of $0.1 \mathrm{~K}$. Two sensors were used to measure the temperature variations in the inlet and outlet tanks, and one sensor was used to measure the room temperature variations. The experiments were carried out at room temperature ( $\sim 297 \mathrm{~K})$. In order to maintain thermal stability during the 
experiments, all heat sources were excluded. The difference between the three temperature sensor measurements was less than $0.3 \mathrm{~K}$. The relative variation of the temperature during the duration of the experiment was less than $0.1 \mathrm{~K}$, and the thermal stability was checked before and during each experiment. The purity of the gases was higher than $99.999 \%$.

The volumes of the inlet and outlet tanks were measured using a simple and accurate method. Using a reference tank with known volume, the ratio between the measured volume and the reference volume was calculated from the mass conservation law. This method was repeated five times, and the maximum deviation of the measured volume values from the mean value was $0.16 \%$.

\section{B. Microchannel characteristics}

Two stainless steel microtubes $T 1$ and $T 2$ (see Table I) were used in this study, and the internal surface of the microtube $T 1$ was coated with Sulfinert. The Sulfinert coating is the leading passivation technique for storage and transfer of low-level organosulfur containing samples. Sulfinert is a silica based coating, completely inert to organosulfur compounds, and was applied to the stainless steel surface by the chemical vapor deposition technique (CVD). Typically, stainless steel adsorbs or reacts with sulfur compounds such as hydrogen sulfide, while the Sulfinert layer prevents sulfur compounds from contacting the reactive stainless steel surface. The low chemical reactivity of the surface, however, is not necessarily related to its attributes, which contribute to momentum and energy transfer.

Both tubes were provided by the RESTEK company, and the details of the fabrication process can be found on the company website (www.restek.com). The internal diameter of the microtubes, as given by the provider, was $250 \mu \mathrm{m}$ though this value was corrected (third column of Table I) by comparing the simulated and measured mass flow rate in the hydrodynamic flow regime, as detailed in Sec. IV. The roughness of the microtube surfaces was not known.

\section{Mass flow rate measurement}

Using the constant volume technique, the mass flow rate through the microtube can be calculated from the equation of state for an ideal gas by assuming steady state conditions as

$$
p V=M \mathcal{R} T,
$$

TABLE I. Dimensions of the microtubes. The internal diameter value of 250 $\mu \mathrm{m}$ was given by the provider and this value was corrected using the measurements in the hydrodynamic flow regime (the third column in the table). The details on the real diameter estimations and their uncertainties are given in Sec. IV.

\begin{tabular}{lccc}
\hline \hline Channel & Material & $D\left[10^{-6} \mathrm{~m}\right]$ & $L\left[10^{-3} \mathrm{~m}\right]$ \\
\hline$T 1$ & Sulfinert & 287.11 & $2340 \pm 1^{\mathrm{a}}$ \\
$T 2$ & Stainless steel & 239.73 & $2013 \pm 1^{\mathrm{a}}$ \\
\hline \hline
\end{tabular}

${ }^{\mathrm{a}}$ For the experiments with $\mathrm{CO}_{2}$ gas, the length of the microtubes $T 1$ and $T 2$ were reduced to $9.96 \pm 0.05$ and $10.33 \pm 0.05 \mathrm{~cm}$, respectively. where $V$ represents the volume of the inlet or outlet tanks, $\mathcal{R}=k / m$ is the specific gas constant; $k$ is the Boltzmann constant; $m$ is the molecular mass; and $p, T$, and $M$ are pressure, temperature, and mass of the gas in a reservoir, respectively. If we assume small variations of the gas properties ( $p$, $M$, and $T$ ) in a tank, Eq. (1) can be written as follows:

$$
\frac{d M}{d t}=\frac{d}{d t}\left(\frac{p V}{\mathcal{R} T}\right)
$$

As was shown in Ref. 9, the relation in Eq. (2) may be transformed into

$$
\frac{d M}{d t}=\frac{V}{\mathcal{R} T} \frac{d p}{d t}(1-\varepsilon), \quad \varepsilon=\frac{d T / T}{d p / p} .
$$

If $\varepsilon$ is small compared to 1 , then the ratio $d M / d t$ can be considered the stationary isothermal mass flow rate $\dot{M}^{\text {exp }}$ through the microtube.

As is clear from Eq. (3), the constant volume technique requires very high thermal stability, which means that the relative temperature variations during the experiment must be much smaller than the relative pressure variations. The conditions in our experiment were such that the deviation of the temperature from its initial value was smaller than $0.1 \mathrm{~K}$ during the duration of the experiment. The relative variation of the temperature, $d T / T$, was on the order of $2 \times 10^{-4}$ compared to $10^{-2}$ for the relative variation of the pressure $d p / p$. Clearly, $\varepsilon$ was less than $2 \times 10^{-2}$ and could be neglected when calculating the mass flow rate. However, the $\varepsilon$ value was added when the uncertainty of the mass flow rate measurements was calculated. Thus, the mass flow rate $\dot{M}^{\text {exp }}$ can be written with the following form:

$$
\dot{M}^{\exp }=\frac{V}{\mathcal{R} T} \frac{d p}{d t} .
$$

The measurement of $\dot{M}^{\text {exp }}$ was affected by a specific relative error of $2 \times 10^{-2}$ due to possible temperature variation during the duration of the experiment, which was included as the term $\Delta T / T$ in Eq. (6).

In the expression for the mass flow rate in Eq. (4), the parameters $V$ and $\mathcal{R}$ were known and remain constant during the experiments. To calculate $d p / d t$ in Eq. (4), we used the recorded data of the pressure variation, $p_{i}$, at different times, $t_{i}$. This variation was on the order of $\pm 1 \%$, and the corresponding data were fitted with a first order polynomial form using the least square method as follows:

$$
p\left(t_{i}\right)=a t_{i}+b
$$

where the slope $a$ of the function $p\left(t_{i}\right)$ is equal to the derivative $d p / d t$, and its value was used in Eq. (4) to calculate the constant experimental mass flow rate.

The uncertainty of the mass flow rate measurement was evaluated using the following expression:

$$
\frac{\Delta \dot{M}^{\exp }}{\dot{M}^{\exp }}=\frac{\Delta V}{V}+\frac{\Delta T}{T}+\frac{\Delta a}{a},
$$


where the uncertainty of the coefficient $a=d p / d t$ was estimated from the standard deviation following the method used in Ref. 9, and its value was found to be less than $0.5 \%$.

The uncertainty of the temperature measurement was very small, but we assumed it to be equivalent to the systematic error made due to the stationary assumption, which was previously evaluated to be $2 \%$. The total uncertainty on the mass flow rate measurement was therefore less than $\pm 4.1 \%$ $(\Delta V / V= \pm 1.6 \%, \Delta T / T= \pm 2 \%$, and $\Delta a / a=0.5 \%)$.

\section{ANALYTICAL SOLUTION IN SLIP REGIME}

The continuum approach based on the Navier-Stokes equation is the widely used for the gas flow simulations. Its application was extended to the slip regime by implementing the first and second order slip boundary conditions for the gas velocity at the wall. ${ }^{19-21} \mathrm{~A}$ brief presentation of the analytical expressions used for the mass flow rate, the velocity slip and tangential momentum accommodation coefficients are given below.

Using the first order slip boundary condition, the mass flow rate through a microchannel with a circular cross section can be expressed in the following form:

$$
\dot{M}=\dot{M}_{P}\left(1+8 \sigma_{p} \frac{1}{\delta_{m}}\right), \quad \dot{M}_{P}=\frac{\pi D^{4} \Delta p p_{m}}{128 \mu \mathcal{R} T L},
$$

where $\dot{M}_{P}$ is the classical Poiseuille mass flow rate, $D$ is the tube diameter, $\Delta p=p_{\text {in }}-p_{\text {out }}$ is the pressure difference, $p_{m}=0.5\left(p_{\text {in }}+p_{\text {out }}\right)$ is the mean pressure, $\mu$ is the viscosity coefficient defined in Eq. (9), ${ }^{22}$ and $\sigma_{p}$ is the viscosity slip coefficient. The rarefaction parameter $\delta$ is defined as

$$
\delta=\frac{p D}{\mu \sqrt{2 \frac{k}{m} T}}
$$

and is based here on the mean pressure $\delta_{m}=\delta\left(p_{m}\right)$. For the viscosity coefficient $\mu$, the expression used is defined in the framework of the variable hard sphere (VHS) model ${ }^{22}$

$$
\mu=\mu_{\text {ref }}\left(\frac{T}{T_{\text {ref }}}\right)^{\omega},
$$

where $\omega$ is the viscosity index and depends on the nature of the gas (see Table II). The reference temperature $T_{\text {ref }}$ is equal to $273.15 \mathrm{~K}$, and the reference values of the viscosity $\mu_{\text {ref }}$ for the different gases are given in Table II. ${ }^{22}$ The rarefaction parameter considered in this section is calculated from the mean

TABLE II. Viscosity coefficient $\mu$, the viscosity index $\omega$, and the $k_{\lambda}$ coefficient [Eq. (10)] according to the VHS model for different gases (Ref. 22).

\begin{tabular}{lccc}
\hline \hline Gas & $\mu_{\text {ref }}\left(10^{-5} \mathrm{~Pa} \cdot \mathrm{s}\right)$ & $\omega$ & $k_{\lambda}$ \\
\hline $\mathrm{He}$ & 1.865 & 0.66 & 0.786 \\
$\mathrm{~N}_{2}$ & 1.656 & 0.74 & 0.731 \\
$\mathrm{Ar}$ & 2.117 & 0.81 & 0.684 \\
$\mathrm{CO}_{2}$ & 1.380 & 0.93 & 0.607 \\
\hline \hline
\end{tabular}

pressure between the two tanks, upstream and downstream of the microtube, and its value ranges from 10 to 4000. This range covers the hydrodynamic and slip flow regimes.

In this work, the authors have chosen the rarefaction parameter $\delta$ in order to characterize the gas flow regime. This choice is made considering the available numerical data in the transitional and the near free molecular flow regimes, presented as a function of the rarefaction parameter in Refs. 23-27. However, originally the definition of various orders in the slip regime is based on the development of the Boltzmann equation dimensionless collisional member following the Knudsen number $K n$ (with $K n<1$ )

$$
K n \sim \frac{1}{\delta}, \quad \text { where } \quad K n=\frac{\lambda}{D}=\frac{k_{\lambda}}{\delta} .
$$

We note that the molecular mean free path $\lambda$ depends on the intermolecular potential through the viscosity and $k_{\lambda}$ coefficients (see Table II), while for the rarefaction parameter, the intermolecular interaction appears only through the viscosity coefficient.

For the velocity slip and accommodation coefficient extraction, the analytical expression for the mass flow rate (7) can be rewritten in a dimensionless form

$$
S=\dot{M} / \dot{M}_{P}=1+8 \sigma_{p} \frac{1}{\delta_{m}}
$$

or in more compact form

$$
S^{T}=B_{0}^{T}+B_{1}^{T} \frac{1}{\delta_{m}}, \quad \text { with } \quad B_{0}^{T}=1, \quad B_{1}^{T}=8 \sigma_{p} .
$$

At this point, the experimental mass flow rate data are normalized according to Eq. (11) and fitted using a form similar to Eq. (12)

$$
S_{f}^{\exp }=B_{0}^{\exp }+B_{1}^{\exp } \frac{1}{\delta_{m}},
$$

where the coefficients $B_{0}^{\exp }$ and $B_{1}^{\text {exp }}$ are the least-square fitting coefficients. ${ }^{12}$ When the velocity slip coefficient is known, notably from identifying $B_{1}^{T}$ and $B_{1}^{\exp }$, then the accommodation coefficient $\alpha$ may be deduced.

The authors of Ref. 28 calculated the values of the velocity slip coefficient using the Bhatnager-Gross-Krook (BGK) kinetic model and the Maxwell diffuse-specular scattering kernel for different values of the accommodation coefficient $\alpha$. They proposed a simple expression associating the velocity slip and the accommodation coefficients, which was later improved in Ref. 26. In the present work, we use the improved expression ${ }^{26}$ to calculate the accommodation coefficient from the experimental values of $\sigma_{p}(\alpha)$

$$
\sigma_{p}(\alpha)=\frac{2-\alpha}{\alpha}\left(\sigma_{p}(1)-0.1211(1-\alpha)\right),
$$

where $\sigma_{p}(1)=1.016$ is the velocity slip coefficient for $\alpha=1$ calculated in Ref. 19, using the BGK kinetic model with the 
assumption of full accommodation of the molecules at the wall.

\section{RESULTS AND DISCUSSION}

\section{A. Hydrodynamic and slip regimes}

The flow of four gases $\left(\mathrm{He}, \mathrm{N}_{2}, \mathrm{Ar}\right.$, and $\mathrm{CO}_{2}$ ) through microtubes with two different surface materials are studied in the rarefaction parameter range [10-4000]. The experimental conditions for both microtubes, $T 1$ and $T 2$, are summarized in Table III. The pressure ratio $\mathcal{P}=p_{\text {in }} / p_{\text {out }}$ between the upstream and downstream tanks is fixed at 1.5 in the hydrodynamic regime, and then it is increased to reach the value of $\sim 4$ in the slip flow regime. It was shown in Refs. 12 and 25 that the nondimensional mass flow rate does not depend significantly on the pressure ratio, and therefore, this change in the pressure ratio does not have a large influence on the extracted velocity slip and accommodation coefficients.

As seen in the previous section, the measured mass flow rate is normalized according to Eq. (11) and is then fitted using the first order in $1 / \delta_{m}$ polynomial form (13), similar to the analytical expression in Eq. (12). From the comparison of the two expressions in Eqs. (12) and (13), the coefficients $B_{i}^{\exp }(i=0,1)$ in Eq. (13) can be expressed with the following form:

$$
B_{0}^{\exp }=1, \quad B_{1}^{\exp }=8 \sigma_{p} .
$$

Nevertheless, the fitting of the experimental data is carried out without fixing the value of the coefficient $B_{0}^{\exp }$ to 1 in Eq. (13), despite the "theoretical value" predicted in Eq. (11). In the hydrodynamic flow regime when the rarefaction parameter tends to infinity, the mass flow rate should be measured equal to the Poiseuille mass flow rate $\dot{M}_{P}$ [see Eq. (7)]. This first fitting is done using the value of the diameter given by the provider. If we suppose that all measured quantities in the Poiseuille expression in Eq. (7) do not have any errors excepting the diameter, then the correct diameter may be calculated from the fitting limit of the measured mass flow rate in the hydrodynamic flow regime, when $\delta_{m}$ tends theoretically to infinity. Thus, canceling the deviation of the coefficient $B_{0}^{\exp }$ from 1 allows us to correct the values of the tube diameter given by the provider, and the tube diameter is chosen to find the Poiseuille mass flow rate limit. The values of the tube diameter as adjusted from the Poiseuille mass flow rate are given in Table I.

After this first step of diameter value adjustment, the measured mass flow rate is fitted again with the polynomial

TABLE III. Experimental conditions.

\begin{tabular}{lcccccc}
\hline \hline Microchannel & & \multicolumn{2}{c}{$T 1$ (Sulfinert) } & & \multicolumn{2}{c}{$T 2(\mathrm{SS})$} \\
\cline { 1 - 3 } \cline { 7 - 8 } \cline { 6 - 7 } Quantity & & Min & Max & & Min & Max \\
\hline Mass flow rate $\dot{M}^{\exp }\left(10^{-12} \mathrm{~kg} / \mathrm{s}\right)$ & 6.5 & 279000 & & 5.4 & 403000 \\
Inlet pressure $p_{\text {in }}(\mathrm{Pa})$ & & 260.8 & 123600 & & 397.3 & 127450 \\
Outlet pressure $p_{\text {out }}(\mathrm{Pa})$ & 77.5 & 78700 & & 108.1 & 80200 \\
Average Knudsen number $K n_{m}$ & $2.2 \cdot 10^{-4}$ & 0.092 & & $2.6 \cdot 10^{-4}$ & 0.090 \\
\hline \hline
\end{tabular}

TABLE IV. Experimental coefficients $B_{0}^{\exp }$ and $B_{1}^{\exp }$ obtained from the first order polynomial fitting for the microtube $T 1$ (Sulfinert).

\begin{tabular}{lccccc}
\hline \hline Gas & $B_{0}^{\exp }$ & $B_{1}^{\text {exp }}$ & $s_{r}$ & $r^{2}$ & $E_{s}$ \\
\hline $\mathrm{He}$ & $0.987 \pm 0.002$ & $9.282 \pm 0.060$ & 0.010 & 0.998 & 0.008 \\
$\mathrm{~N}_{2}$ & $0.988 \pm 0.001$ & $10.181 \pm 0.062$ & 0.007 & 0.998 & 0.006 \\
$\mathrm{Ar}$ & $0.998 \pm 0.002$ & $10.140 \pm 0.087$ & 0.011 & 0.997 & 0.009 \\
$\mathrm{CO}_{2}$ & $1.005 \pm 0.002$ & $8.906 \pm 0.060$ & 0.007 & 0.999 & 0.006 \\
\hline \hline
\end{tabular}

form in the range [10-4000]. The obtained fitting coefficients and the statistical characteristics of the fitting procedure such as the determination coefficient, $r^{2}$, the standard error, $E_{s}$, and the squared residual sum, $s_{r}$, are given in Tables IV and $\mathrm{V}$, for the tubes $T 1$ and $T 2$, respectively. The squared residual sum, $s_{r}$, is defined as following: $s_{r}=\sqrt{\frac{1}{n-k} \sum_{i=1}^{n} e_{i}^{2}}$, where $n$ is the number of the measured points; $e_{i}=S_{i}^{\exp }-S_{f_{i}}^{\exp }$ is the local difference between the measured values $S_{i}^{\text {exp }}$, normalized according to Eq. (11), and the fitted values $S_{f_{i}}^{\exp }$ [Eq. (13)]; $k$ is the number of the unknown coefficients of the fitting model, and in our case, $k=2$. The standard error is $E_{s}=\sqrt{\frac{1}{n} \sum_{i=1}^{n} e_{i}^{2}} / S_{m}^{\exp }$, and here, $S_{m}^{\exp }$ is the average value of the measured quantities.

As is clear from the results presented in Tables IV and V, the procedure to adjust the value of the tube diameter works well. The values of the $B_{0}^{\exp }$ coefficients for all gases are now very close to their theoretical values of one, even if the diameter value adjustment using the Poiseuille expression is carried out only for Ar. The deviation of the coefficient $B_{0}^{\exp }$ from 1 for all gases does not exceed $1 \%$. Finally, let us note that by using the hydrodynamic regime for the diameter calibration, we minimize the surface effects and also the unknown roughness effects. Moreover, the Poiseuille expression is not modified by the roughness, and a correct "effective hydraulic diameter" can be determined in any case.

From Tables IV and V, one can see that the values of the determination coefficients, $r^{2}$, are very close to 1 , and those of the residual variance, $s_{r}$, are smaller than 0.012 , which confirms the quality of fitting for both microtubes $T 1$ and $T 2$. The maximum value of the standard error made on the fittings is smaller than $1 \%$. Therefore, by analyzing the statistical characteristics of the fittings, we can conclude that the fitting expressions of first order terms of $1 / \delta_{m}$ accurately represent the experimental data.

TABLE V. Experimental coefficients $B_{0}^{\exp }$ and $B_{1}^{\exp }$ obtained from the first order polynomial fitting for the microtube $T 2$ (SS).

\begin{tabular}{lccccc}
\hline \hline Gas & $B_{0}^{\exp }$ & $B_{1}^{\exp }$ & $s_{r}$ & $r^{2}$ & $E_{s}$ \\
\hline $\mathrm{He}$ & $0.992 \pm 0.001$ & $9.659 \pm 0.041$ & 0.006 & 0.999 & 0.005 \\
$\mathrm{~N}_{2}$ & $0.992 \pm 0.001$ & $9.641 \pm 0.049$ & 0.006 & 0.999 & 0.005 \\
$\mathrm{Ar}$ & $1.002 \pm 0.002$ & $9.994 \pm 0.092$ & 0.012 & 0.997 & 0.010 \\
$\mathrm{CO}_{2}$ & $0.994 \pm 0.002$ & $9.679 \pm 0.063$ & 0.010 & 0.998 & 0.009 \\
\hline \hline
\end{tabular}


TABLE VI. Experimental accommodation and slip coefficients for microtube $T 1$ (Sulfinert).

\begin{tabular}{lccc}
\hline \hline Gas & Molar mass $(\mathrm{g} / \mathrm{mol})$ & $\sigma_{p}$ & $\alpha$ \\
\hline $\mathrm{He}$ & 4.00 & $1.160 \pm 0.008$ & $0.930 \pm 0.003$ \\
$\mathrm{~N}_{2}$ & 28.02 & $1.273 \pm 0.008$ & $0.881 \pm 0.003$ \\
$\mathrm{Ar}$ & 39.95 & $1.268 \pm 0.011$ & $0.883 \pm 0.004$ \\
$\mathrm{CO}_{2}$ & 44.01 & $1.113 \pm 0.008$ & $0.951 \pm 0.004$ \\
\hline \hline
\end{tabular}

The values of the velocity slip coefficient, $\sigma_{p}$, are given in Tables VI and VII for both tubes. This "experimental" velocity coefficient is obtained from the fitting coefficient $B_{1}^{\exp }$ using Eq. (15). The standard error of the coefficient $\sigma_{p}$ in Tables VI and VII derives from the fitting error on the coefficients $B_{1}^{\exp }$ and does not take into account the systematic uncertainty coming from the uncertainties on the mass flow rate measurements [see Eq. (6)], and the uncertainties related to the Poiseuille expression in Eq. (7). Globally, we see that the values of the experimental velocity slip coefficients (see Tables VI and VII) are different from those obtained theoretically in Ref. $19\left(\sigma_{p}=1.016\right)$, showing that the accommodation is not complete in our experimental conditions.

To analyze the gas properties present in both microtubes $T 1$ and $T 2$ in the range of the rarefaction parameter [10-4000], we must first recall some results obtained in our previous studies. We observed in previous measurements $9,12,29,30$ that, for monoatomic gases, an increase in the molecular weight causes a decrease of the TMAC. Furthermore, we noted that in the restricted case of monoatomic gases, the weight and spherical molecule size are directly related. This led us to suggest that a large spherical size could increase the molecule specular reflection by minimizing the effects of the wall asperity or of the wall structure (roughness and atomic arrangement). The present results again confirm this tendency for monoatomic gases under consideration, where again we see that a larger size of spherical monoatomic molecules leads to a smaller value of the accommodation coefficient at the wall.

In addition, the present results show that for the polyatomic molecules the specular mass effect at the wall is very moderate $\left(\mathrm{N}_{2}\right.$ case $)$ or largely inverted $\left(\mathrm{CO}_{2}\right.$ case $)$. The nonspherical structure of the polyatomic molecules associated with the corresponding internal degrees of freedom seems to favor a random direction of reflection and, consequently, a diffusive reflection. Furthermore, this diffusive effect is larger for more complex molecular structures, as seen by comparing the results in Tables VI and VII for $\mathrm{CO}_{2}$ and $\mathrm{N}_{2}$.

TABLE VII. Experimental accommodation and slip coefficients for microtube T2 (SS).

\begin{tabular}{lccc}
\hline \hline Gas & Molar mass $(\mathrm{g} / \mathrm{mol})$ & $\sigma_{p}$ & $\alpha$ \\
\hline $\mathrm{He}$ & 4.00 & $1.212 \pm 0.005$ & $0.907 \pm 0.002$ \\
$\mathrm{~N}_{2}$ & 28.02 & $1.205 \pm 0.006$ & $0.909 \pm 0.003$ \\
$\mathrm{Ar}$ & 39.95 & $1.249 \pm 0.012$ & $0.891 \pm 0.005$ \\
$\mathrm{CO}_{2}$ & 44.01 & $1.210 \pm 0.008$ & $0.907 \pm 0.003$ \\
\hline \hline
\end{tabular}

For completeness, we report that the length of the microtubes $T 1$ and $T 2$ were reduced by a factor of 20 when the experiments were carried out for the carbon-dioxide gas, and therefore the experimental conditions for this gas were different. In the $\mathrm{CO}_{2}$ experiment, the low pressure measurements of the pressure variations in both tanks become difficult to be measured with certainty. Therefore, the tube length was decreased to increase the mass flow rate, and consequently, the pressure variations in the tanks [see Eqs. (4) and (19)]. We believe this length change does not have a significant influence on the interacting law and the accommodation process.

Comparing the specific results obtained in each tube, we observe that in microtube $T 1$ the highest TMAC values are obtained for $\mathrm{He}$ and $\mathrm{CO}_{2}$, and the lowest values for $\mathrm{N}_{2}$ and Ar (see Table VI).

In the microtube $T 2$, however, the stainless steel internal surface tends to be less diffusive for $\mathrm{He}$ and $\mathrm{CO}_{2}$ than the $T 1$ sulfinert surface (see Table VII). Consequently, the values of TMAC obtained in the $T 2$ microtube with the stainless steel internal surface lie in a narrower range [0.891-0.909], centered close to 0.9, when compared with the $T 1$ internal surface results. These different accommodation coefficient values for different tubes may be explained by the different materials and roughness of the respective surfaces. An additional element governing these value differences may be found by considering the chemical reactive character of the stainless steel surface, because contact with the stainless steel $T 2$ surface submits each gas to a physical or chemical influence, and the gas interacting behavior tends toward the same limit whatever its initial nature.

In Table VIII, a comparison of our results with the accommodation coefficients obtained by other authors ${ }^{11,29,31}$ is given. All experiments are carried out in microtubes, but with different diameters varying from 25 to $100 \mu \mathrm{m}$. The data treatment was also slightly different in that the second order term in the $1 / \delta$ development in the range [3-4000] was implemented in Refs. 29 and 31, with only the first order implemented in Ref. 11 and in the present study. Despite the differences in the experimental setups and the data treatment used in this study and those of Refs. 29 and 31, a similar behavior of the TMAC value, which decreases with increasing molecular mass for monoatomic gases, is observed, though in Ref. 11, the opposite behavior is found.

The different values of TMAC in Table VIII show a high dependence upon the surface material, though the roughness of the surface may also play an important role. Unfortunately, the values of the roughness for the tubes used in the present experiments are not known.

The development of a second order approach in the rarefaction parameter range [3-4000] is attempted, but the experimental data obtained in this range are neither sufficiently numerous nor homogeneously distributed to allow a pertinent statistical treatment. Thus, we cannot extract the theoretical coefficient (velocity slip and accommodation coefficients) with sufficient quantitative reliability. The required accuracy is all the more important here because our 
TABLE VIII. TMAC obtained from the present experiments and from Refs. 11, 29, and 31.

\begin{tabular}{lcccc}
\hline \hline & Material & Helium & Nitrogen & Argon \\
\hline Porodnov et al. (Ref. 11) & Glass & $0.895 \pm 0.004$ & $0.925 \pm 0.014$ & $0.927 \pm 0.028$ \\
Ewart et al. (Ref. 29) & Silica & $0.986 \pm 0.009$ & $0.981 \pm 0.041$ & $0.942 \pm 0.017$ \\
Perrier et al. (Ref. 31) & Silica & $1.00 \pm 0.019$ & $0.961 \pm 0.005$ & $0.954 \pm 0.010$ \\
Present results (T1) & Sulfinert & $0.930 \pm 0.003$ & $0.881 \pm 0.003$ & $0.883 \pm 0.004$ \\
Present results (T2) & Stainless steel & $0.907 \pm 0.002$ & $0.909 \pm 0.003$ & $0.891 \pm 0.005$ \\
\hline
\end{tabular}

investigation shows qualitatively a very significant second order in this range.

\section{B. Transitional and near free molecular flow regimes}

The transitional and free molecular flow regimes are the most complicated regimes from the experimental point of view. In these regimes, in addition to the problems of leakage and outgassing (see Sec. II A), the detection of flow parameters and especially the pressure variation in the tanks becomes difficult and requires highly sensitive pressure transducers. For example, for the nominal outlet tank pressure of $14.2 \mathrm{~Pa}$, the pressure varies about $0.28 \mathrm{~Pa}$ during the experimental time of $t=250 \mathrm{~s}$. In our experimental setup, we use an extremely sensitive pressure transducer with a full scale resolution of $1.5 \times 10^{-5}$ and a response time of $30 \mathrm{~ms}$.

In contrast to the slip flow regime for the transitional regime, an analytical expression for the mass flow rate does not exist in the literature. Numerical results for the mass flow rate obtained by using different kinetic models may be found in Refs. 23-27.

In this paper, we use numerical results for the mass flow rate in the transitional and free molecular flow regimes obtained from the linearized S-model kinetic equation ${ }^{27}$ for diffuse reflection $(\alpha=1)$ and for diffuse-specular reflection $(\alpha$ is different from 1) at the wall. The solution of the linearized kinetic equation is obtained under two main assumptions: first, the tube diameter $D$ is small compared to the tube length $L(D \ll L)$; and second, the dimensionless pressure gradient along the tube is small compared to one. The second assumption is not satisfied in our experiments, because the pressure ratio between inlet and outlet tanks varies from 1.5 to 4 . However, in Ref. 25, it was shown that the linearization of the kinetic equation may be done under the less restrictive condition of

$$
\frac{D}{L} \frac{p_{\text {in }}-p_{\text {out }}}{p_{m}} \ll 1, \quad \text { for } \quad \delta_{m} \leq 1 .
$$

This restriction is fulfilled in our experimental conditions, which allows us to use a portion of the results obtained in Ref. 27, where the numerical values of the dimensionless mass flow rate

$$
G_{P}(\delta)=-\frac{\sqrt{2 \frac{k}{m} T}}{\pi(D / 2)^{3} d p / d z} \dot{M}
$$

are given as a function of the rarefaction parameter $\delta$, defined by Eq. (8). To obtain a dimensionless mass flow rate characterizing each experiment, however, we cannot directly apply the results of Ref. 27 using Eq. (17) because the rarefaction parameter $\delta$ and the pressure vary along the channel. To take into account the rarefaction parameter variations along the tube, we can integrate Eq. (17) along the microtube, as proposed in Ref. 25

$$
G=\frac{1}{\delta_{\text {out }}-\delta_{\text {in }}} \int_{\delta_{\text {in }}}^{\delta_{\text {out }}} G_{P}(\delta) d \delta,
$$

where $\delta_{\text {in }}$ and $\delta_{\text {out }}$ are the values of the rarefaction parameter corresponding to the pressure in the inlet and outlet tanks, respectively. The dimensionless mass flow rate $G$ is related to the dimensional rate in the following way:

$$
G=\frac{L \sqrt{2 \frac{k}{m} T}}{\pi(D / 2)^{3}\left(p_{\text {in }}-p_{\text {out }}\right)} \dot{M} .
$$

The approach described above successfully implemented in Ref. 29, where the comparison with a simpler approximate formula $^{25}$

$$
G=G_{P}\left(\frac{\delta_{\text {in }}+\delta_{\text {out }}}{2}\right)=G_{P}\left(\delta_{m}\right),
$$

was also fulfilled and a very good agreement between the results obtained from Eqs. (18) and (20) was found. Therefore, in the present paper, we compare the experimental results $G^{\exp }$ in the dimensionless form from Eq. (19) with the numerical results derived from Ref. 27, where the influence of the pressure and $\delta$ variation along the axis are taken into account according to Eq. (20). In this way, we deduce the most appropriate "experimental" value of the accommodation coefficient.

\section{Results in the transitional regime}

The dimensionless mass flow rates (experimental and numerical) through the microtubes $T 1$ and $T 2$ are presented in Figs. 2 and 3, respectively, as a function of the mean rarefaction parameter $\delta_{m}$, calculated according to Eq. (8) for the mean value of the pressure between the inlet and outlet tanks. The minimum values of the mean rarefaction parameters reached experimentally are different for each of the working gases ( $\mathrm{He}, \mathrm{N}_{2}$, and $\mathrm{Ar}$ ). This difference is essentially due to the difference in the molecular mass of each gas, so that for the same value of pressure, the rarefaction parameter for the lighter He gas is approximately 3 times 


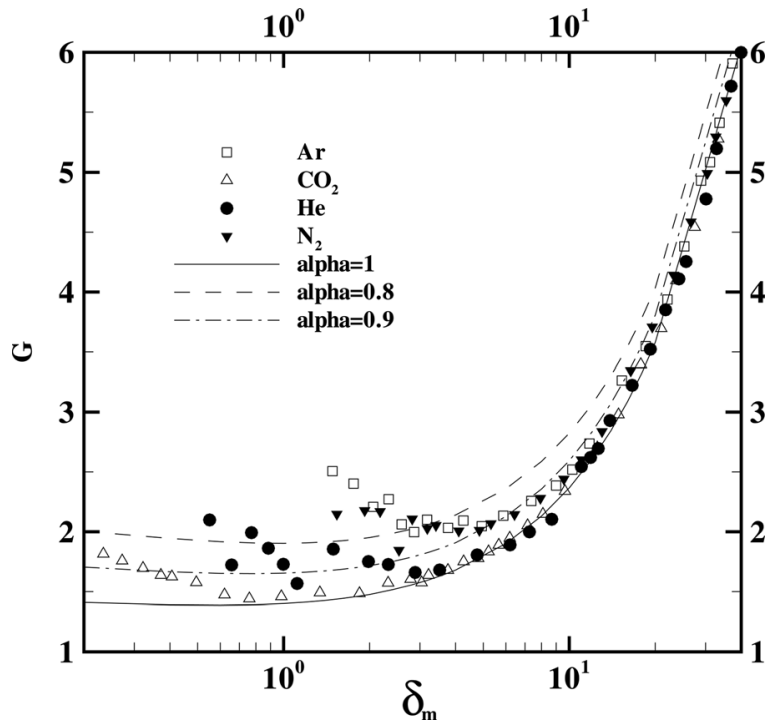

FIG. 2. Dimensionless mass flow rate $G$ as function of the mean rarefaction parameter $\delta_{m}$ for microtube $T 1$.

smaller than for Ar. This trend changes in the case of $\mathrm{CO}_{2}$, however, where the smallest rarefaction parameter is on the same order as He, especially for the $T 2$ tube, even though its molecular mass is 11 times greater. This is due to the reduced length of the tubes (see Table I) used in the $\mathrm{CO}_{2}$ measurements, which allows the mass flow rate through the tube to remain detectable for the smallest $\mathrm{CO}_{2}$ outlet pressure values.

The analysis of the experimental data in the entire range of the rarefaction parameters may be explained in several steps. The first step is the choice of a fitting curve form. We propose to fit the numerical data of the reduced mass flow rate, $G$, obtained from the numerical data, given in Ref. 27, using the procedure described in Ref. 25 and pointed out in Eq. (20), in the form



FIG. 3. Dimensionless mass flow rate $G$ as function of the mean rarefaction parameter $\delta_{m}$ for microtube $T 2$.

$$
\begin{aligned}
F\left(\delta_{m}\right)= & A_{1} \delta_{m}^{2}+A_{2} \delta_{m}+A_{3}+A_{4} \frac{1}{\delta_{m}}+A_{5} \frac{1}{\delta_{m}^{0.5}} \\
& +A_{6} \frac{1}{\delta_{m}^{0.1}}+A_{7} \frac{1}{\delta_{m}^{0.01}}
\end{aligned}
$$

where the rarefaction parameter varies from 0.001 to 100 . The accuracy of this interpolation calculated as

$$
\sqrt{\sum_{i}\left(F\left(\delta_{m}\right)-G_{P}\left(\delta_{m}\right)\right)^{2}}
$$

does not exceed 1\%. The numerical data in Ref. 27 are available only for $\alpha=0.8$ and 1 . We obtain the fitting curves for the intermediate values of the accommodation coefficient of $0.85,0.875,0.9,0.925,0.95$, and 0.975 using a linear interpolation between the results calculated for $\alpha=0.8$ and 1 for a fixed value of $\delta_{m}$. In this way, we acquire the fitting results for the reduced mass flow rate corresponding to eight accommodation coefficients. The second step is fitting the experimental data of each gas using the form of data representation shown in Eq. (21) to obtain the $A_{i}^{\exp }$ coefficients

$$
\begin{aligned}
F^{\exp }\left(\delta_{m}\right)= & A_{1}^{\exp } \delta_{m}^{2}+A_{2}^{\exp } \delta_{m}+A_{3}^{\exp }+A_{4}^{\exp } \frac{1}{\delta_{m}} \\
& +A_{5}^{\exp } \frac{1}{\delta_{m}^{0.5}}+A_{6}^{\exp } \frac{1}{\delta_{m}^{0.1}}+A_{7}^{\exp } \frac{1}{\delta_{m}^{0.01}} .
\end{aligned}
$$

The numerical results corresponding to the eight accommodation coefficients acquired by the Eq. (21) fit are integrated over the entire $\delta_{m}$ range. Similarly, the fit of the experimental data for each gas using Eq. (23) is integrated over the same $\delta_{m}$ range. Finally, we compare the "experimental integral" value of each gas with all eight "numerical integrals." In this way, we determine which accommodation coefficient value is closest to each gas experimental data value, and thereby we find the "experimental" accommodation coefficient of each gas, (see Table IX). As we can conclude from Table IX, the accommodation coefficients found in the slip flow regime and in the whole experimental range (from hydrodynamic to the transitional flow regimes) are globally in good agreement.

The experimental data points of $\mathrm{N}_{2}$ and Ar obtained for the microtube $T 1$ (see Fig. 2) are similar and are situated higher than the data points for $\mathrm{He}$ and $\mathrm{CO}_{2}$, which confirms the results obtained in the slip regime, showing that the

TABLE IX. Experimental accommodation coefficients for microtubes $T 1$ (Sulfinert) and $T 2$ (stainless steel) obtained in the slip regime $\left(\alpha^{1}\right)$, and in the entire rarefaction range $\left(\alpha^{2}\right)$.

\begin{tabular}{lccccc}
\hline \hline & \multicolumn{2}{c}{$T 1$} & & \multicolumn{2}{c}{$T 2$} \\
\cline { 2 - 3 } \cline { 5 - 6 } Gas & $\alpha^{1}$ & \multicolumn{1}{c}{$\alpha^{2}$} & & $\alpha^{1}$ & $\alpha^{2}$ \\
\hline $\mathrm{He}$ & $0.930 \pm 0.003$ & $0.975 \pm 0.025$ & & $0.907 \pm 0.002$ & $0.95 \pm 0.025$ \\
$\mathrm{~N}_{2}$ & $0.881 \pm 0.003$ & $0.90 \pm 0.025$ & & $0.909 \pm 0.003$ & $0.90 \pm 0.025$ \\
$\mathrm{Ar}$ & $0.883 \pm 0.004$ & $0.90 \pm 0.025$ & & $0.891 \pm 0.005$ & $0.90 \pm 0.025$ \\
$\mathrm{CO}_{2}$ & $0.951 \pm 0.004$ & $0.975 \pm 0.025$ & & $0.907 \pm 0.003$ & $0.95 \pm 0.025$ \\
\hline \hline
\end{tabular}


accommodation coefficients are lower for $\mathrm{Ar}$ and $\mathrm{N}_{2}$ than for $\mathrm{He}$ and $\mathrm{CO}_{2}$.

The experimental data points of all gases in the microtube $T 2$ (see Fig. 3), obtained for $\delta_{m}>8$, lie between the curves for $\alpha=0.9$ and 1 . When $\delta_{m}$ is less than 8 , a significant deviation is observed for $\mathrm{N}_{2}$ and $\mathrm{Ar}$, but for $\mathrm{He}$ and $\mathrm{CO}_{2}$ the experimental data points continue to follow the same curve, $\alpha=0.9$. This is in agreement with the results found in the continuum analysis for $T 2$, where the accommodation coefficient was found in a very narrow range for all gases. The results shown here again confirm a similar TMAC value for the $\mathrm{He} / \mathrm{CO}_{2}$ couple and the $\mathrm{N}_{2} / \mathrm{Ar}$ couple. Moreover, we again find a lower TMAC value for the $\mathrm{N}_{2} / \mathrm{Ar}$ couple in the most rarefied domain.

The values found with the analysis in the transitional regime using the S-model kinetic equation are slightly higher than the values found in the continuum regime. Nevertheless, the analysis made in the transitional regime globally confirms the qualitative and quantitative results of TMAC found with the continuum approach, and therefore the same TMAC value can be generally applied in all flow regimes.

\section{CONCLUSION}

The experimental investigation of the flow through circular microchannels is presented. The constant volume technique is used to measure the mass flow rates of the gases (helium, nitrogen, argon, and carbon-dioxide).

The continuum approach (Navier-Stokes equations) associated with the first order velocity slip boundary condition is used in the slip flow regime to obtain the experimental velocity slip and the tangential momentum accommodation coefficients based on the Maxwell diffuse-specular kernel.

The results for the slip and accommodation coefficients are compared to the results obtained by other authors.

The transitional and near free molecular regimes are also investigated experimentally and the experimental results obtained are compared with a linearized S-model kinetic equation for different TMAC at the wall.

A first attempt to obtain the values of the accommodation coefficients in the entire range of the gas rarefaction is undertaken. The results show a good agreement globally of the two different approaches for the TMAC extraction. Consequently, the results for the accommodation coefficient are similar for the entire range of the gas rarefaction.

For monoatomic molecules, the accommodation coefficients increase when the molecular mass decreases. In addition, for the polyatomic molecule, the molecular structure complexity seems to cause the accommodation coefficient to increase.

The effect of the surface material on the gas-solid surface interaction is highlighted by comparing the values of the accommodation coefficients of two surface materials
(Sulfinert and stainless steel). In the $T 2$ tube, the various TMAC values lie in a narrower range than in the Sulfinert tube (T1), and this change is obtained through a decreasing diffusivity of the reflection for $\mathrm{He}$ and $\mathrm{CO}_{2}$. Otherwise, the stainless steel (T2) tends to reduce significantly the specificity of the different gases in the gas-surface reflection.

\section{ACKNOWLEDGMENT}

This research received funding from the European Community's Seventh Framework Program (ITNFP7/2007-2013) under grant agreement number 215504.

${ }^{1}$ C. Cercignani and M. Lampis, Transport Theory Stat. Phys. 1, 101 (1971).

${ }^{2}$ S. K. Dadzie and J. G. Méolans, J. Math. Phys. 45, 1804 (2004).

${ }^{3}$ O. V. Sazhin, S. F. Borisov, and F. Sharipov, J. Vac. Sci. Technol. A 19, 2499 (2001).

${ }^{4}$ A. Agrawal and S. V. Prabhu, J. Vac. Sci. Technol. A 26, 634 (2008).

${ }^{5}$ S. Colin, P. Lalonde, and R. Caen, Heat Transf. Eng. 25, 23 (2004).

${ }^{6}$ J. Harley, Y. Huang, H. Bau, and J. Zemel, Fluid Mech. 284, 257 (1995).

${ }^{7}$ J. Maurer, P. Tabeling, P. Joseph, and H. Willaime, Phys. Fluids 15, 2613 (2003).

${ }^{8}$ Y. Zohar, S. Y. K. Lee, W. Y. Lee, L. Jiang, and P. Tong, Fluid Mech. 472, 125 (2002).

${ }^{9}$ T. Ewart, P. Perrier, I. A. Graur, and J. G. Méolans, Exp. Fluids 41, 487 (2006).

${ }^{10}$ E. B. Arkilic, K. S. Breuer, and M. A. Schmidt, Fluid Mech. 437, 29 (2001).

${ }^{11}$ B. T. Porodnov, P. E. Suetin, S. F. Borisov, and V. D. Akinshin, J. Fluid Mech. 64, 417 (1974).

${ }^{12}$ T. Ewart, P. Perrier, I. A. Graur, and J. G. Méolans, Fluid Mech. 584, 337 (2007).

${ }^{13}$ J. Pitakarnnop, S. Varoutis, D. Valougeorgis, S. Geoffroy, L. Baldas, and S. Colin, Microfluid. Nanofluid. 8, 57 (2010).

${ }^{14}$ M. Hadj Nacer, I. Graur, and P. Perrier, Houille Blanche 49 (2011).

${ }^{15}$ H. Yamaguchi, T. Hanawa, O. Yamamoto, Y. Matsuda, Y. Egami, and T. Niimi, Microfluid. Nanofluid. 11, 57 (2011).

${ }^{16}$ K. Jousten, G. Messer, and D. Wandrey, Vacuum 44, 135 (1993).

${ }^{17}$ L. Peksa, T. Gronych, M. Vicar, M. Jerab, P. Repa, J. Tesar, D. Prazak, and Z. Krajicek, Measurement 44, 1143 (2011).

${ }^{18}$ L. Wangkui, Z. Dixin, L. Qiang, L. Shiliang, L. Detian, and M. Yang, Vacuum 47, 519 (1996).

${ }^{19}$ C. Cercignani and A. Daneri, Phys. Fluids 6, 993 (1963).

${ }^{20}$ G. Karniadakis and A. Beskok, Microflows: Fundamentals and Simulation (Springer, New York, 2002).

${ }^{21}$ I. A. Graur, J. G. Méolans, and D. E. Zeitoun, Microfluid. Nanofluid. 2, 64 (2006).

${ }^{22}$ G. A. Bird, Molecular Gas Dynamics and the Direct Simulation of Gas Flows (Oxford University Press Inc., New York, 1994).

${ }^{23}$ B. T. Porodnov and F. T. Tuchvetov, J. Eng. Phys. 36, 61 (1979).

${ }^{24}$ S. S. Lo and S. K. Loyalka, J. Appl. Math. Phys. 33, 419 (1982).

${ }^{25}$ F. Sharipov and V. D. Seleznev, J. Vac. Sci. Technol. A 12, 2933 (1994).

${ }^{26}$ F. Sharipov and V. Seleznev, J. Phys. Chem. Ref. Data 27, 657 (1998).

${ }^{27}$ F. Sharipov, J Therm. Sci. Technol. A 14, 2627 (1996).

${ }^{28}$ S. K. Loyalka, N. Petrellis, and S. T. Stvorick, Phys. Fluids 18, 1094 (1975).

${ }^{29}$ T. Ewart, P. Perrier, I. A. Graur, and J. G. Méolans, Microfluid. Nanofluid. 3, 689 (2007).

${ }^{30}$ I. A. Graur, P. Perrier, W. Ghozlani, and J. G. Méolans, Phys. Fluids 21, 102004 (2009).

${ }^{31}$ P. Perrier, I. A. Graur, T. Ewart, and J. G. Méolans, Phys. Fluids 23, 042004 (2011). 